\title{
Low Birth Weight and Maternal and Neonatal Deaths are Complications of Systemic Lupus Erythematosus in Pregnant Pristane Induced Lupus Mice
}

\author{
Handono KALIM, ${ }^{1}$ Kusworini HANDONO, ${ }^{2}$ Mirza Zaka PRATAMA, ${ }^{3}$ Sofi Nur FITRIA, ${ }^{3}$ Vardian MAHARDIKA ${ }^{3}$ \\ ${ }^{1}$ Department of Internal Medicine, Faculty of Medicine Brawijaya University, Division of Rheumatology, Malang, Indonesia \\ ${ }^{2}$ Department of Clinical Pathology, Faculty of Medicine Brawijaya University, Malang, Indonesia \\ ${ }^{3}$ Department of Biomedical Science, Faculty of Medicine Brawijaya University, Malang, Indonesia
}

\begin{abstract}
Objectives: This study aims to investigate the relationship between systemic lupus erythematosus and pregnancy in pristane induced lupus mice model, including the pregnancy outcome for mothers and neonates.

Materials and methods: Sixteen female Balb/c mice 6-8 week-old were separated into two groups: pristane induced lupus (PIL) mice group that received a single intraperitoneal injection of $0.5 \mathrm{ml}$ of pristane and healthy control (HC) group that received phosphate-buffered saline injection. At one month after injection, all mice were mated with 8-10 week-old male Balb/c mice. All mice from both groups were observed for body weight and survival. On the day of births; number of neonates born, birth weight, and length of neonates were calculated. Neonates were also monitored for survival rate and their ages along the study.

Results: There was no statistically significant difference in body weight before and after delivery, survival rate during pregnancy, number of neonates born, and gestational ages of mothers from both groups. Mothers began to die in the postpartum period. On third day after delivery, $0 \%$ of mothers from PIL group survived whereas $100 \%$ mothers from HC group survived $(p=0.000)$. Of neonates born from PIL group, $15 \%$ survived at the end of fifth day after birth whereas $100 \%$ of neonates from HC group still survived $(p=0.000)$. Neonates from PIL group also had significantly lower body weight and body length at birth compared to HC group ( $p=0.000$ and $p=0.000$, respectively).

Conclusion: Maternal and neonatal death, low birth weight, and low birth length are complications of systemic lupus erythematosus at pregnancy in pristane induced lupus mice model.

Keywords: Low birth weight and length; maternal and neonatal deaths; pregnancy; systemic lupus erythematosus.
\end{abstract}

Systemic lupus erythematosus (SLE) is an autoimmune disease with multiple organs manifestations including the kidneys, joints, hematopoietic organs, and nervous system. Immune system aberrations, as well as heritable, hormonal and environmental factors interplay in the expression of organ damage and clinical manifestation of the disease. ${ }^{1}$ SLE predominantly affects women of childbearing age. Although patients with SLE are as fertile as women in the general population, their pregnancies may be associated with complications. Pregnancy and its outcome is a major concern to most SLE patients.
Queries regarding the risk of disease flares during pregnancy and chance of fetal loss are often raised. ${ }^{2,3}$

Pregnant patients with SLE have an increased risk complication of pregnancy. The incidence of pre-eclampsia in lupus pregnancies ranges from 5 to $38 \%$ in various reported series, which is higher than that of pregnancies in women without SLE. ${ }^{4,5}$ Recent studies have provided information regarding the course of pregnancy in patients with lupus nephritis. Hypertension developed frequently (37-56\%) during pregnancies in these patients. Pre-eclampsia may develop in up to 
$30 \%$ of SLE patients with preexisting nephritis., ${ }^{1,4}$ Other researchers also found that women with SLE had a higher risk to develop opportunistic infections such as pneumonia or sepsis which also contribute to maternal death during pregnancy. ${ }^{6,7}$

Fetal outcome of SLE also have high risk complication. Pregnancies in SLE patients are characterized by an increased incidence of fetal wastage (abortions and stillbirths), prematurity, and intrauterine growth retardation. ${ }^{1,4}$ The rates of abortion and stillbirths in lupus pregnancies vary from $6-35 \%$ and $0-22 \%$, respectively, which are higher than that of the general population. ${ }^{4}$ Active lupus nephritis, previous history of fetal death, and the presence of the antiphospholipid antibodies have been shown to be predictive factors for fetal wastages in lupus pregnancies. ${ }^{5}$ Rahman et al. ${ }^{8}$ in their study suggested that active renal disease is a significant predictor for fetal loss $(p<0.012)$. Maternal hypertension was also identified as a significant statistical predictor for prematurity and intrauterine growth retardation.

Neonates that come from a mother with SLE have a high risk of neonatal lupus erythematosus syndrome. Neonatal lupus erythematosus is a syndrome consisting of congenital heart block, transient cutaneous lupus lesions, cytopenia, hepatic, and other systemic manifestations in children born to mothers with SLE, Sjögren's syndrome, or other rheumatic diseases with a positive anti-Ro or anti-La antibodies. ${ }^{1,9}$

Limited studies have been conducted to monitor the risk of SLE in pregnancy and the fetal outcome. Low incidence ratio, ethical problems, and unwillingness to participate in studies still constitute obstacles. Thus, in this study, we aimed to investigate the relationship between SLE and pregnancy in pristane induced lupus mice model, including the pregnancy outcome for mothers and neonates.

\section{MATERIALS AND METHODS}

All in vivo studies were undertaken at Pharmacology Laboratory of Brawijaya University Research Center. The studies and animal care have been approved by ethical committee of Brawijaya University with related codes of practice. Female virgin Balb/c mice (obtained from Pusvetma Surabaya, East Java, Indonesia) aged 6-8 weeks were housed under standard conditions and provided with food and water ad libitum. Mice were habituated to the holding room for a minimum of one week before undergoing experimental procedures.

Mice were separated into two groups: pristane induced lupus (PIL) group which received a single intraperitoneal injection of $0.5 \mathrm{~mL}$ of pristane $(2,6,10$, 14-tetramethylpentadecane) and healthy control (HC) group which received phosphate-buffered saline injection in a different cage. ${ }^{10}$

After four weeks injection of pristane, female Balb/c mice were mated with 8 to 14 weeks old $\mathrm{Balb} / \mathrm{c}$ mice with a male to female ratio of $1: 2$ on each cage. Females were inspected daily for vaginal plugs; sighting a vaginal plug was designated as day 0 of pregnancy. All mice from both groups were observed weekly for body weight and survival until day of birth and daily from birth until five days after birth.

To prevent biases, all neonates born were cared by foster mother. On the day of births; number of neonates born, birth weight and length were calculated. Neonates were also monitored for survival rate and their ages along the study.

\section{Statistical analysis}

Unpaired t-test was used to compare the differences between clinical characteristics of mothers, maternal and neonatal ages, and birth weight and length of neonates. Chi-square or Fisher's exact test was used to compare the differences between survival rate of mothers and neonates. Data were described as mean \pm standard deviation. Statistical analysis was performed using PASW version 17.0 software program (SPSS Inc., Chicago, IL, USA).

\section{RESULTS}

From both groups, we used homogenous samples of mice which had similar characteristics to ensure that there were no biases in this research. As shown in Table 1, there were no differences in the clinical characteristics of both groups before pregnancy. In both HC and PIL groups, 
Table 1. Clinical characteristics of mothers from healthy control and pristane induced lupus groups

\begin{tabular}{|c|c|c|c|c|c|}
\hline \multirow[t]{2}{*}{ Variable } & \multicolumn{2}{|c|}{ HC group $(n=8)$} & \multicolumn{2}{|c|}{ PIL group $(n=8)$} & \multirow[b]{2}{*}{$p^{*}$} \\
\hline & $\%$ & Mean \pm SD & $\%$ & Mean \pm SD & \\
\hline Baseline maternal ages (weeks) & \multirow{6}{*}{100} & $6.5 \pm 0.9$ & \multirow{6}{*}{100} & $6.8 \pm 0.9$ & 0.552 \\
\hline Maternal prepartum body weight (gr) & & $27.7 \pm 2.9$ & & $27.7 \pm 2.8$ & 0.199 \\
\hline Maternal survival rate during pregnancy & & & & & - \\
\hline Maternal postpartum body weight (gr) & & $39.5 \pm 1.5$ & & $40.1 \pm 1.1$ & 0.364 \\
\hline Number of neonates born & & $9.6 \pm 1.8$ & & $10.3 \pm 1.9$ & 0.516 \\
\hline Gestation time (days) & & $20.6 \pm 1.8$ & & $20.3 \pm 1.3$ & 0.644 \\
\hline
\end{tabular}

baseline maternal ages $[6.50 \pm 0.926$ weeks in HC group vs. $6.75 \pm 0.886$ weeks in PIL group; $p=0.552$, confidence interval (CI) 95\%] and maternal pre partum body weight $(27.72 \pm 2.911$ grams in HC group vs. $27.69 \pm 2.81$ grams in PIL group; $p=0.199$, CI 95\%) were not statistically significantly different (Table 1).

We also monitored the survival rate of mothers from first day of pregnancy until delivery. We detected that all of mice in both groups survived $100 \%$. As shown in Table 1 , there were no differences between $\mathrm{HC}$ and PIL groups in terms of maternal postpartum body weights $(39.50 \pm 1.512$ grams in HC group vs. $40.12 \pm 1.126$ grams in PIL group; $p=0.364$, CI 95\%). At birth, number of neonates born from both groups were also not statistically significantly different $(9.62 \pm 1.847$ in $\mathrm{HC}$ group vs. $10.25 \pm 1.909$ in PIL group; $p=0.516$, CI 95\%). Gestation time between two groups also did not differ statistically significantly (20.62 \pm 1.847 days in HC group vs. $20.25 \pm 1.282$ days in PIL group; $\mathrm{p}=0.644$, CI 95\%).

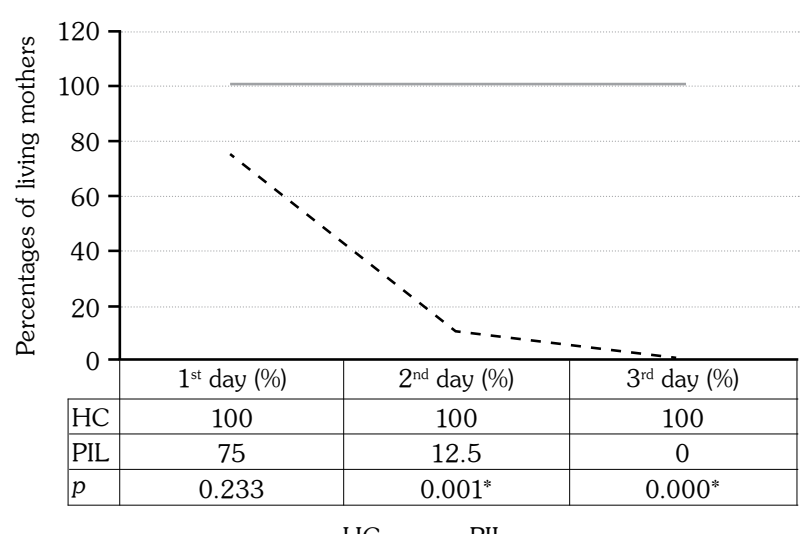

Figure 1. Survival rate of mothers from both healthy control (HC) and pristane induced lupus (PIL) groups from first day until third day after delivery. * Values are significantly different between groups $(p<0.05)$.
After the delivery, all mothers were monitored for survival rate. Figure 1 shows the maternal survival rates from first day until third day postpartum. On the first day postpartum, we found that all mothers from $\mathrm{HC}$ group survived whereas only $75 \%$ of mothers from PIL group survived although the difference was not statistically significant ( $p=0.233$, CI 95\%). A statistically significant difference was seen between two groups from second day postpartum with only $12.5 \%$ of mothers from PIL group surviving whereas all mice from $\mathrm{HC}$ group still survived ( $p=0.001$, CI 95\%). On third day postpartum, all mothers from PIL group died, whereas $100 \%$ of mothers from HC group still survived $(p=0.000$, CI 95\%).

From these data, we were able to measure the average age of mothers after the delivery, as shown in Figure 2. We showed that all mothers from $\mathrm{HC}$ group survived to the age of more than five days postpartum whereas average age from

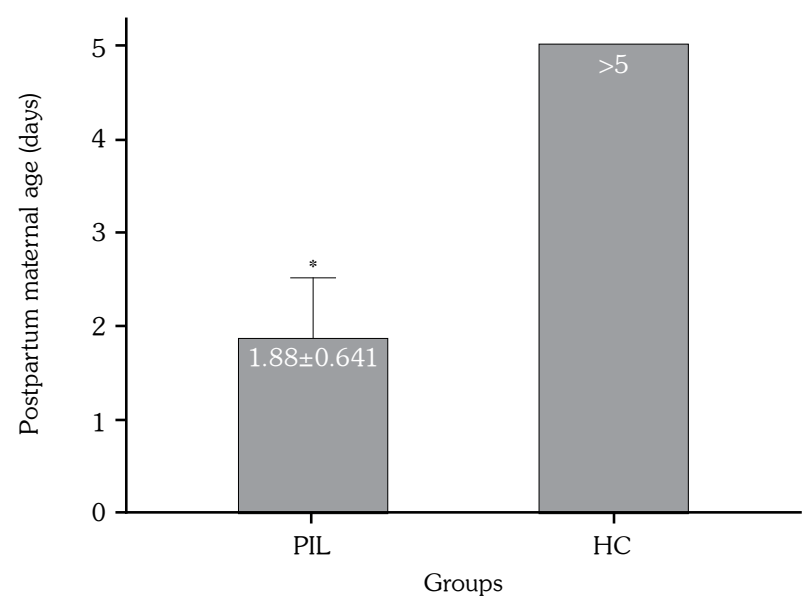

Figure 2. Average ages of mothers from both pristane induced lupus (PIL) and healthy controls (HC) groups after delivery. Ages were counted from delivery until maternal deaths. "Values are significantly different compared with HC group. 


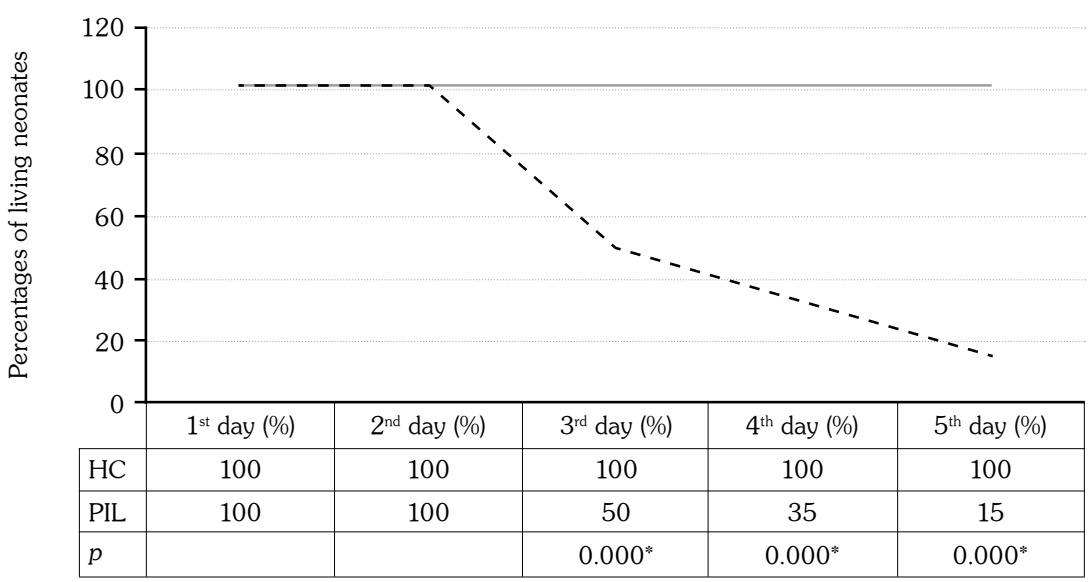

Figure 3. Survival rates of neonates from both healthy control $(\mathrm{HC})$ and Pristane induced lupus (PIL) groups from first day until fifth day after delivery. *Values are significantly different between groups $(p<0.05)$.

PIL group was only $1.88 \pm 0.641$ days postpartum $(p=0.000$, CI 95\%).

All neonates born were monitored daily for their survival rates, as shown in Figure 3. We detected that until second day of birth, all mice from both $\mathrm{HC}$ and PIL groups survived. On the third day of birth, $50 \%$ of neonates from PIL group were dead whereas $100 \%$ of neonates from $\mathrm{HC}$ group still survived and the difference was statistically significant between the groups $(p=0.000$, CI 95\%). On the fourth day of birth, only $35 \%$ of neonates from PIL group survived whereas all neonates from $\mathrm{HC}$ group still survived

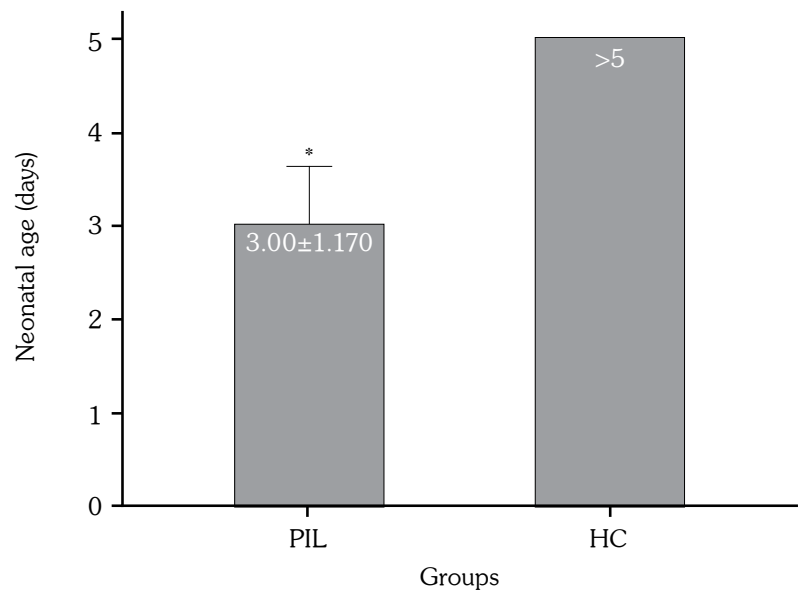

Figure 4. Average ages of neonates from both pristane induced lupus (PIL) and healthy controls (HC) groups. Ages were counted from delivery until neonatal deaths. *Values are significantly different between groups $(p<0.05)$. $(p=0.000$, CI 95\%). At the end of fifth day of birth, neonates which still survived from PIL group were only $15 \%$ whereas all neonates from HC group still survived ( $p=0.000$, CI 95\%).

From these data, we were able to measure the average age of neonates after birth, as shown in Figure 4. We found that all neonates from $\mathrm{HC}$ group survived until the age of more than five days whereas average age of neonates from PIL group was only $3.00 \pm 1.170$ days $(p=0.002$, CI 95\%).

Neonates born from HC mothers had slightly larger size in appearance than neonates born from PIL group. Aside from the sizes, we did not detect any macroscopic abnormalities or differences between two groups. On the day of birth, all neonates from both groups were assessed for birth weight and length. Table 2 shows the differences of birth weight and length between two groups. We noted that birth weights in PIL group were significantly lower

Table 2. Birth weights and lengths of neonates from healthy control and pristane induced lupus groups

\begin{tabular}{lcccc}
\hline Variable & HC group $(\mathrm{n}=20)$ & & PIL group $(\mathrm{n}=20)$ & \\
\cline { 2 - 2 } \cline { 5 - 5 } & Mean $\pm \mathrm{SD}$ & & Mean \pm SD & $p^{*}$ \\
\hline Birth weight $(\mathrm{gr})$ & $1.6 \pm 0.1$ & & $1.4 \pm 0.0$ & 0.000 \\
Birth length $(\mathrm{cm})$ & $3.9 \pm 0.1$ & & $3.7 \pm 0.1$ & 0.000
\end{tabular}

HC: Healthy control; PIL: Pristane induced lupus; SD: Standard deviation; * Statistically significant, $\mathrm{p}<0.05$; CI $\% 95$. 
compared to HC group $(1.563 \pm 0.037$ grams in HC group vs. $1.145 \pm 0.031$ grams in PIL group; $p=0.000$, CI 95\%). Also, birth lengths of neonates measured from cranial-caudal were significantly shorter in PIL group compared to $\mathrm{HC}$ group as seen in Table $2(3.868 \pm 0.046 \mathrm{~cm}$ in $\mathrm{HC}$ group vs. $3.699 \pm 0.067 \mathrm{~cm}$ in PIL group; $\mathrm{p}=0.000$, CI 95\%).

\section{DISCUSSION}

Although the outcome of pregnancy in patients with SLE has improved considerably in recent years, complications involving neonates and mothers with SLE remain to be a main topic for researches. In this study, we showed that the survival rate of mothers with SLE in PIL mice group decreased significantly compared to the healthy ones. Our research indicated that none of the mothers in PIL group survived whereas 100\% of healthy mice were still alive until third day of postpartum period. In addition, the average age of mothers in PIL group was only $1.88 \pm 0.644$ days whereas all mice mothers and neonates from $\mathrm{HC}$ group were alive until more than five days.

This result was similar with results of other researches published previously in which maternal death was a major complication in pregnant women with SLE. ${ }^{1,4}$ There are many facts and theories which may explain the cause of death in pregnant mothers with SLE. Clowse et al. ${ }^{6}$ suggested that women with SLE had a two to four-fold increased rate of pregnancy complications, such as preeclampsia and hematologic complications, than the non-SLE population. In addition, it was shown that the risk of maternal death was 20-fold higher than the non-SLE population and the cause of death was indicated as the increased risk of medical complications in pregnant women with SLE, such as sepsis and pneumonia. It was also demonstrated that pregnant mothers with SLE tend to have higher risk to get flares which may affect many organs and cause death. ${ }^{2,3}$ Lupus nephritis and infections (mainly opportunistic disease) were suggested to be major causes of deaths in pregnant women with active SLE flares. ${ }^{11}$

Flares of lupus during pregnancy are generally attributed to the progressive increase in serum estrogen levels during pregnancy, especially in the third semester. Estrogens augment immunologic reactivity and this phenomenon are often cited as the basis to the increased risk of autoimmunity such as SLE in pregnant women. ${ }^{3}$ Many studies suggested that estrogens can regulate the cytokine gene expression in different cell types, such as interleukin 6 (IL)-6, IL-10, IL-12, and IL-2. ${ }^{12,13}$ A recent study also showed that estrogen increased the IL-4 production while decreasing IL-2 and interferon gamma secretion by T cells. ${ }^{14}$ IL-4 is a cytokine which plays a key role in $\mathrm{T}$ helper 2 differentiation where $\mathrm{T}$ helper 2 is a major component which can contribute to the development of lupus nephritis..$^{15}$ Another possible mechanism for the increasing of flare during pregnancy may be the decreased Treg function in decidua and peripheral blood in pregnant women. ${ }^{16}$

In our study, all mother deaths due to SLE occurred in postpartum period, not during pregnancy period. We hypothesized that maternal deaths in postpartum period might be the result of the accumulation of tissue organ damage which occurred during pregnancy until the time of delivery or something happened in mothers' body after delivery. A national study done by Clowse et al. $^{6}$ showed that hematologic complications are common among pregnant SLE women. Anemia was diagnosed in over $12 \%$ of SLE pregnancies at the time of delivery. Risk of venous thromboembolism was five to eight-fold higher and the risk of stroke was 6.5-fold higher for women with SLE compared to other women after delivery. Similar results showed that postpartum hemorrhage and fever postpartum were significantly higher in mothers associated with SLE. Furthermore, it was demonstrated that mothers with SLE had a higher risk to develop opportunistic infection and sepsis during postpartum period. ${ }^{7}$

During postpartum period, levels of cortisol are at highest level. ${ }^{17}$ Cortisol is a powerful and important modulator of the immune system. Cortisol can suppress the activity of immune system by many ways. While it was shown that low levels of cortisol might contribute to a higher disease activity in SLE, other researches stated that higher levels of cortisol was associated with significantly higher mortality due to septic shock. Higher incidence of fever and sepsis that may develop during postpartum period might be due 
to this high level of cortisol. A stress condition due to disease activity of SLE might also contribute to increased cortisol levels. ${ }^{18,19}$

We also detected that not only mothers but also neonates born from mice with SLE suffered from serious complications. Survival rate of neonates born from mothers with SLE was significantly lower compared to healthy ones. Also, neonates born from mothers with SLE had significantly lower birth weight and length. These results are compatible to results obtained in infants born from human mothers with SLE. The frequencies of spontaneous abortion and stillbirths increased in women with SLE, with the stillbirth rate nearly five times greater than for non-lupus pregnancies. ${ }^{20}$ Another research showed that neonates born from mothers with SLE were likely to deliver premature and smaller babies. ${ }^{4,7}$ In addition, newborn resuscitation for $>3$ minutes, respiratory distress syndrome, assisted ventilation, patent ductus arteriosus were more common in newborns from mothers with SLE which associated with newborn deaths after delivery. ${ }^{7}$ Preterm births may occur in approximately 20\% of pregnancies with SLE which was also associated with neonatal complications. However, in this study, we did not detect any differences for the time of delivery between the two groups. Finally, neonatal lupus erythematosus, which occurs via placental passage of maternal anti-Ro/SSA and anti-La/SSB antibodies which bind to fetal tissue and cause either congenital heart block or noncardiac neonatal lupus, ${ }^{9}$ is a condition that may develop in neonates born from mothers with SLE.

Fetal growth restriction may result from several factors, including disease activity, hypercoagulability and placental pathology. Impaired fetal growth happens when blood flow through the placenta is restricted by placental pathology. Placental pathology in pregnant women with SLE is characterized by ischemia/hypoxia, decidual vasculopathy, decidual and fetal thrombi, chronic villitis, decreased placental weight and placental infarctions along with deposit of fibrin, immunoglobulin $G$, immunoglobulin $\mathrm{M}$, immunoglobulin $\mathrm{A}$, and complement 3 in the trophoblastic membrane. ${ }^{22,23}$ Hypercoagulability may also result in placental infarctions and consequent fetal hypoxia. This condition may develop due to many factors, such as sticky platelet syndrome, pregnancy-induced protein $\mathrm{S}$ deficiency, increased inflammatory factors related to SLE disease activity, hyperhomocysteinemia due to folate deficiency, and antiphospholipid antibodies. ${ }^{1,24}$

In conclusion, this study has shown the complications of pregnancy in mouse models of SLE, reporting some facts regarding the high mortality rates of mothers and neonates and also low birth weight and length of neonates born from mothers with SLE. These conditions may also develop in poorly treated human mothers with SLE. However, their causes are still not clearly understood. Autopsy and histopathological studies, which were not performed in the present study, may help to detect these causes. Further research is needed to fully understand the placental pathology and also the cause of death in pregnancies with SLE. Understanding the cause of death, pathology, and mechanism involving SLE in mothers and infants may help researchers to comprehend the pathogenesis of SLE during pregnancy and also assist physicians to determine how to treat pregnant SLE patients to control possible future complications.

\section{Acknowledgements}

We thank Dr. drh. Sri Murwani, MP for guiding us for keeping the animal models in laboratory. We also thank Brawijaya University and DIKTI for providing fund for this research.

\section{Declaration of conflicting interests}

The authors declared no conflicts of interest with respect to the authorship and/or publication of this article.

\section{Funding}

This research was funded by Direktorat Jendral Perguruan Tinggi Indonesia (DIKTI) via Penelitian Unggulan Perguruan Tinggi 2014 research program.

\section{REFERENCES}

1. Dhar JP, Sokol RJ. Lupus and pregnancy: complex yet manageable. Clin Med Res 2006;4:310-21.

2. Ruiz-Irastorza G, Lima F, Alves J, Khamashta MA, Simpson J, Hughes GR, et al. Increased rate of lupus flare during pregnancy and the puerperium: 
a prospective study of 78 pregnancies. Br J Rheumatol 1996;35:133-8.

3. Stojan G, Baer AN. Flares of systemic lupus erythematosus during pregnancy and the puerperium: prevention, diagnosis and management. Expert Rev Clin Immunol 2012;8:439-53.

4. Cortés-Hernández J, Ordi-Ros J, Paredes F, Casellas M, Castillo F, Vilardell-Tarres M. Clinical predictors of fetal and maternal outcome in systemic lupus erythematosus: a prospective study of 103 pregnancies. Rheumatology (Oxford) 2002;41:643-50.

5. Mok CC, Wong RW. Pregnancy in systemic lupus erythematosus. Postgrad Med J 2001;77:157-65.

6. Clowse ME, Jamison M, Myers E, James AH. A national study of the complications of lupus in pregnancy. Am J Obstet Gynecol 2008;199:1-6.

7. Nili F, McLeod L, O'Connell C, Sutton E, McMillan D. Maternal and neonatal outcomes in pregnancies complicated by systemic lupus erythematosus: a population-based study. J Obstet Gynaecol Can 2013;35:323-8.

8. Rahman P, Gladman DD, Urowitz MB. Clinical predictors of fetal outcome in systemic lupus erythematosus. J Rheumatol 1998;25:1526-30.

9. Buyon JP, Clancy RM. Neonatal lupus: review of proposed pathogenesis and clinical data from the US-based Research Registry for Neonatal Lupus. Autoimmunity 2003;36:41-50.

10. Chowdhary VR, Grande JP, Luthra HS, David CS. Characterization of haemorrhagic pulmonary capillaritis: another manifestation of Pristane-induced lupus. Rheumatology (Oxford) 2007;46:1405-10.

11. Ritchie J, Smyth A, Tower C, Helbert M, Venning M, Garovic V. Maternal deaths in women with lupus nephritis: a review of published evidence. Lupus 2012;21:534-41.

12. Liu J, Beller DI. Distinct pathways for NF-kappa B regulation are associated with aberrant macrophage IL-12 production in lupus- and diabetes-prone mouse strains. J Immunol 2003;170:4489-96.

13. Katsiari CG, Tsokos GC. Transcriptional repression of interleukin-2 in human systemic lupus erythematosus. Autoimmun Rev 2006;5:118-21.
14. Xia X, Zhang S, Yu Y, Zhao N, Liu R, Liu K, et al. Effects of estrogen replacement therapy on estrogen receptor expression and immunoregulatory cytokine secretion in surgically induced menopausal women. J Reprod Immunol 2009;81:89-96.

15. Miyake K, Akahoshi M, Nakashima H. Th subset balance in lupus nephritis. $\mathrm{J}$ Biomed Biotechnol 2011;2011:980286.

16. Sasaki Y, Sakai M, Miyazaki S, Higuma S, Shiozaki A, Saito S. Decidual and peripheral blood CD4+CD25+ regulatory $\mathrm{T}$ cells in early pregnancy subjects and spontaneous abortion cases. Mol Hum Reprod 2004; 10:347-53.

17. Meinlschmidt G, Martin C, Neumann ID, Heinrichs M. Maternal cortisol in late pregnancy and hypothalamicpituitary-adrenal reactivity to psychosocial stress postpartum in women. Stress 2010;13:163-71.

18. Sam S, Corbridge TC, Mokhlesi B, Comellas AP, Molitch ME. Cortisol levels and mortality in severe sepsis. Clin Endocrinol (Oxf) 2004;60:29-35.

19. Shah D, Kiran R, Wanchu A, Bhatnagar A. Relationship between $\mathrm{T}$ lymphocyte subsets and cortisol in systemic lupus erythematosus. Kathmandu Univ Med J (KUMJ) 2009;7:213-9.

20. Dhar JP, Essenmacher LM, Ager JW, Sokol RJ. Pregnancy outcomes before and after a diagnosis of systemic lupus erythematosus. Am J Obstet Gynecol 2005;193:1444-55.

21. Clark CA, Spitzer KA, Nadler JN, Laskin CA. Preterm deliveries in women with systemic lupus erythematosus. J Rheumatol 2003;30:2127-32.

22. Magid MS, Kaplan C, Sammaritano LR, Peterson M, Druzin ML, Lockshin MD. Placental pathology in systemic lupus erythematosus: a prospective study. Am J Obstet Gynecol 1998;179:226-34.

23. Ogishima D, Matsumoto T, Nakamura Y, Yoshida K, Kuwabara Y. Placental pathology in systemic lupus erythematosus with antiphospholipid antibodies. Pathol Int 2000;50:224-9.

24. Andersen JC, Dhar P, Essenmacher L, Ager J, Sokol R. Determinants of thrombophilia in an urban systemic lupus erythematosus population. Blood 2005;106 [Abstract 4134]. 